\title{
Differential Proper-Motion Measurements of The Cygnus Egg Nebula: The Presence of Equatorial Outflows
}

\author{
Toshiya Ueta and Rachael L. Tomasino \\ Department of Physics and Astronomy, MS 6900, University of Denver, Denver, CO 80208 \\ and \\ Brian A. Ferguson ${ }^{1}$ \\ Space Telescope Science Institute, Baltimore, MD 21218
}

Received __; accepted _ _

\footnotetext{
${ }^{1}$ Currently: Des Moines University Medical School
} 


\begin{abstract}
We present the results of differential proper-motion analyses of the Egg Nebula (RAFGL 2688, V1610 Cyg) based on the archived two-epoch optical data taken with the Hubble Space Telescope. First, we determined that the polarization characteristics of the Egg Nebula is influenced by the higher optical depth of the central regions of the nebula (i.e., the "dustsphere" of $\sim 10^{3}$ AU radius), causing the nebula illuminated in two steps - the direct starlight is first channeled into bipolar cavities and then scattered off to the rest of the nebula. We then measured the amount of motion of local structures and the signature concentric arcs by determining their relative shifts over the 7.25-yr interval. Based on our analysis, which does not rely on the single-scattering assumption, we concluded that the lobes have been excavated by a linear expansion along the bipolar axis for the past $\sim 400 \mathrm{yr}$, while the concentric arcs have been generated continuously and moving out radially at about $10 \mathrm{~km} \mathrm{~s}^{-1}$ for the past $\sim 5,500 \mathrm{yr}$, and there appears to be a colatitudinally-increasing trend in the radial expansion velocity field of the concentric arcs. There exist numerical investigations into the mass-loss modulation by the central binary system, which predict such a colatitudinally-increasing expansion velocity field in the spiral-shock trails of the mass-loss ejecta. Therefore, the Egg Nebula may represent a rare edge-on case of the binary-modulated circumstellar environs, corroborating the previous theoretical predictions.
\end{abstract}

Subject headings: Stars: AGB and post-AGB — Stars: individual (Egg Nebula) — Stars: mass-loss - Circumstellar matter — Proper motions 


\section{Introduction}

The Cygnus Egg Nebula (V1610 Cyg, RAFGL 2688; hereafter, the Egg Nebula) is one of the first infrared sources discovered with an optical bipolar nebulosity (Nev et al. 1975). This object, usually referred to as a proto-planetary nebula (Kwok 1993; Van Winckel 2003), consists of the central star in the post-asymptotic giant branch (post-AGB) phase and the physically-detached circumstellar shell, which is a consequence of mass loss during the preceding AGB phase. While AGB mass loss is qualitatively understood dust-driven, the exact physical mechanisms of the structure formation in the circumstellar shell are still not understood completely.

The Egg Nebula is known for its signature bipolar lobes and rather circular concentric $\operatorname{arcs}($ Sahai et al. 1998b). While there are about a dozen sources with such arcs, the Egg Nebula is known to possess the largest number of arcs (>20; Hrivnak et al. 2001; Su 2004). These concentric arcs are qualitatively perceived as manifestations of some kind of mass loss modulations that took place during the early AGB phase. However, the deduced temporal intervals do not match with any of the known theoretical temporal intervals (e.g., thermal pulsing and surface pulsations of AGB stars; Su 2004).

One of the direct methods to understand the dynamics of the circumstellar structure formation is to perform a differential proper-motion analysis and measure the amount of relative translational motion of specific shell structures. We performed such an analysis for the bipolar lobes and the equatorial structures of the Egg Nebula using NICMOS imaging-polarimetry data taken with a 5.5-yr baseline and found that (1) the lobes expanded linearly (i.e., the expansion velocity linearly proportional to the distance to the expansion center), (2) the distance to the Egg Nebula was $420 \pm 60$ pc, and (3) the object (star and nebula) itself experienced its own proper motion at $(13.7 \pm 2.0,10.2 \pm 2.0){\mathrm{mas} \mathrm{yr}^{-1}}^{-1}$ (Ueta et al. 2006, 2012). However, we were unable to measure differential proper-motion 
of the concentric arcs because the NICMOS field of view was too small and the surface brightnesses were too weak is in the near-IR.

Balick et al. (2012) performed the latest attempt using ACS/HRC and WFC3/UVIS images with a 6.65-yr baseline. They reported (1) a uniform expansion of the bipolar lobes by $2.5 \%$ and (2) radial motion of the arcs by $0^{\prime \prime} 07$. However, their analysis was limited to the central $\sim 20^{\prime \prime}$ and lacked background reference sources to properly align multi-epoch images. Hence, they were unable to confirm the bulk motion of the nebula and assumed that the shell structures expanded symmetrically.

In this work, we performed differential proper-motion measurements of the concentric arcs using the archived imaging-polarimetry data from WFPC2 and ACS/WFC, exploiting the data set's largest field of view, longest exposure time, and longest temporal baseline to overcome the deficiencies of the previous analyses. Below, we describe the data set and reduction procedure $(\S 2)$, present the reduced imaging polarimetry data, differential proper-motion measurements, and results of our analysis (§3), and summarize our finding $(\S 4)$.

\section{Observations and Data Reduction}

We used the archived data of the Egg Nebula in the F606W band with polarizers taken with WFPC2 (GTO/WF2-6221; PI: J. Trauger) on 1995 July 17 (epoch 1, hereafter) and ACS/WFC (CAL/ACS-9586; PI: W. Sparks) on 2002 October 16 (epoch 2, hereafter), which provide a 7.25-yr baseline (Table 11). We reduced the data using the PyRAF/STSDAS MultiDrizzle package version 3.13 (Fruchter et al. 2009) 1 to process the multi-program data into a common frame of reference. After removing cosmic-ray hits from the pipeline-

\footnotetext{
${ }^{1} \mathrm{PyRAF}$ and STSDAS are products of the STScI, which is operated by AURA for NASA.
} 
calibrated data using L.A.Cosmic (van Dokkum 2001; there was only one exposure with each of the three WFPC2 polarizers), we performed two rounds of MultiDrizzle processes to refine the image alignment by using background point sources as spatial anchors.

In the end, we generated two sets of three polarizer images for a $60^{\prime \prime} \times 60^{\prime \prime}$ field at the $0{ }^{\prime} .05 \mathrm{pix}^{-1}$ scale centered at the catalog coordinates of the Egg Nebula $\left(21^{\mathrm{h}} 02^{\mathrm{m}} 18.27\right.$, $+36^{\circ} 41^{\prime} 37^{\prime \prime}$.0; Sahai et al. 1998a). Finally, these polarizer images were combined into two sets of Stokes images via matrix transformations for each of the WFPC2 and ACS polarizers as described by Biretta \& McMaster (1997) and Gonzaga et al. (2011), respectively.

We measured the amount of shift for each background source and rejected those that shifted more than two- $\sigma$ of the distribution: after this exercise 31 sources were adopted as stationary spatial anchors. Because the Egg Nebula was imaged near the center of the instrument arrays (central $80^{\prime \prime} \times 80^{\prime \prime}$ for WFPC2/WF and $200^{\prime \prime} \times 200^{\prime \prime}$ for ACS/WFC) these anchors are not affected by the geometric distortion, which may contribute to false proper motions near the aperture edges. The accuracy of alignment was assessed by a residual vector root-mean-square offset computed from the Gaussian centroid positions of these 31 spatial anchors and determined to be as good as 0.36 pix (0".018).

\section{Data Analysis and Discussion}

\subsection{Photometric Variability}

The Stokes I images (Figs. 1 $19, b)$ are the total flux images at the Broad V band in the AB magnitude system (Oke 1974; Gonzaga et al. 2011). These maps show the signature bipolar lobes plus the searchlight beams as well as the concentric arcs. The two-epoch aperture photometry revealed that the total integrated flux of the Egg Nebula decreased by $36 \%$ over 7.25 yrs, while the individual fluxes of the $\mathrm{N}$ and $\mathrm{S}$ lobes decreased by $34 \%$ and 
$41 \%$, respectively (Table 2).

Balick et al. (2012) reported similar but smaller flux variations, with the $\mathrm{N}$ lobe faded more than the S lobe (by $11 \%$ and 3\%, respectively), over 6.65-yr baseline immediately following ours. Moreover, Hrivnak et al. (2010) discovered that the integrated flux of the $\mathrm{N}$ lobe varies with a roughly 90-day period. These observations suggest that both $\mathrm{N}$ and S lobes vary their fluxes periodically with a phase lag, which is probably a manifestation of the pulsation of the central star modulated by the time-delay due to the line-of-sight path-length difference between the two lobes of the inclined nebula.

\subsection{Polarization Characteristics}

\subsubsection{Polarized-Flux-Only Maps}

The linearly-polarized-flux-only images, $I_{\mathrm{pol}}$, were constructed with the Stokes $Q$ and $U$ images $\left(I_{\mathrm{pol}}=\sqrt{Q^{2}+U^{2}} ;\right.$ Figs. 11, $\left.\mathrm{d}\right)$. In the single-scattering limit, $I_{\mathrm{pol}}$ images represent the cross-sections of the circumstellar shell because $I_{\text {pol }}$ tends to be the strongest when the angle of scattering is close to $90^{\circ}$. This uniqueness of $I_{\text {pol }}$ had been used previously to probe the dust density structure in the evolve star circumstellar shells (Ueta et al. 2005, 2007).

Contrary to our expectations, however, the bipolar lobes and searchlight beams appeared more prominently than the concentric arcs (Figs. 1k,d). This indicates that the nebula cannot be approximated in the single-scattering limit (as evidenced by the presence of the dust lane): the Egg Nebula is therefore illuminated by a two-step process, in which starlight is first directed into the lobes and then scattered off from the lobes to the rest of the nebula. 


\subsubsection{Polarization Strength Maps}

The polarization strength images, $P=I_{\mathrm{pol}} / I$, display the relative strength of polarization more clearly (Figs. 1, f, f). While stronger polarizations ( $\gtrsim 40 \%)$ are seen in the lobes and searchlight beams, weaker polarizations $(\lesssim 40 \%)$ are dominant along the equatorial plane except for the region of medium polarizations (about 30 to $50 \%$, delineating the central $2.4^{\prime \prime} \times 3.8^{\prime \prime}$ region around the dust lane $\left(1.0 \times 10^{3}\right.$ by $1.6 \times 10^{3} \mathrm{AU}$ at $\left.420 \mathrm{pc}\right)$.

This medium-polarization region appears to represent the surface of a marginal central dust concentration (often referred to as a dust cocoon; Latter et al. 1993; Sahai et al. 1998a.b; Goto et al. 2002) at which the line-of-sight optical depth at $V$ becomes greater than unity (i.e., dustsphere as in photosphere and MOLsphere). This dustsphere and the bipolar lobes are essentially brightly-lit surfaces that illuminate the rest of the nebula. Hence, dust-scattered light from the rest of the nebula tends to be de-polarized, i.e., weak in $I_{\mathrm{pol}}$ and $P$.

The structure of this central dust concentration is still unknown. Cox et al. (2000) reported the presence of a hollow $\mathrm{CO}$ shell of $1^{\prime \prime}$ radius expanding at $\sim 10 \mathrm{~km} \mathrm{~s}^{-1}$ at the position angle of $\sim 54^{\circ}$ (coincident with the orientation of the $1.3 \mathrm{~mm}$ dust continuum). Thus, this medium-polarization-strength region could represent the "near-side surface" of this central expanding $\mathrm{CO} /$ dust shell. To better assess the geometry of the central material distribution, further investigations at higher spatial resolution at optically-thin bands are required.

\subsubsection{Polarization Angle Maps}

The polarization angle $\left(P A=\frac{1}{2} \arctan (U / Q)\right)$ images show the position angle of the electric vector of the incident light (Figs. $1 \mathrm{~g}, \mathrm{~h})$. The PA images for optically-thin nebulae 
typically show a pattern of an azimuthal gradient, which represents continuously changing polarization vector angles with respect to the position of the illumination source. However, our maps reveal roughly the same angle near the center generally aligned with the dust lane $\left(107^{\circ}\right.$ of $\mathrm{E}$ of $\left.\mathrm{N}\right)$, indicating higher dust density around the dust lane at which the single-scattering approximation breaks down. For this reason, we were unable to pinpoint the location of the illumination source by back-tracing the polarization vectors (e.g., Weintraub \& Kastner 1993; Weintraub et al. 2000; Ueta et al. 2006) more accurately than a one- $\sigma$ error ellipse of $3.4^{\prime \prime} \times 1.8^{\prime \prime}$.

\subsection{Differential Proper-Motion Measurements}

\subsubsection{Nebula Expansion}

As discussed above, the optical reflection nebulosity of the Egg Nebula is caused by a two-step illumination process in rather de-polarized light. Therefore, we reverted to the total intensity $I$ maps (i.e., to utilise all the flux available to us) to follow the differential proper-motion of local shell structures using the same correlational method as in our previous analysis. Briefly, this method measures the amount of translational shift of a given structure between two epochs via a cross-correlation analysis between cutouts of the two-epoch images (e.g., Currie et al. 1996; Morse et al. 2001). We use our own IDL script set created as part of our previous proper-motion investigations of the Egg Nebula in the near-IR (Ueta et al. 2006 for details).

To make the analysis more tractable with the $I$ maps, we edge-enhanced the shell structure while minimizing the background noise by processing the maps with the Roberts' Cross operator (Roberts 1963). These maps are shown in Fig. 2. Because concentric arcs are azimuthally symmetric, the present correlational method is unable to break the azimuthal 
degeneracy and uniquely trace translational shifts of arc segments along the azimuthal direction. This prevented us from following the differential proper-motion of structures azimuthally away from the bipolar axis, and hence, from independently confirming the bulk motion of the nebula and location of the expansion center. Thus, for the present analysis we adopted the previously discovered (1) bulk motion of the Egg Nebula at a rate of $(13.7 \pm 2.0,10.2 \pm 2.0)$ mas $\mathrm{yr}^{-1}$, (2) the location of the illumination source/expansion center, and (3) the distance to the Egg Nebula at $420 \pm 60$ pc as found in our previous analysis (Ueta et al. 2006).

We then selected 30 distinct local structures that register more than five $\mathrm{S} / \mathrm{N}$ with respect to the sky background distributed in the lobes (13 structures) and searchlight beams radially beyond the lobes (17 structures) and performed the correlational analysis to measure proper motion of these structures. To disregard the inadvertent azimuthal shifts, we extracted only the projected radial component of the expansion of the structures as presented in Fig. 33.

The same data are displayed as a plot of the projected radial component of the differential proper-motion vector $\left(v_{\text {rad }}\right.$ in mas $\mathrm{yr}^{-1}$ and $\left.\mathrm{km} \mathrm{s}^{-1}\right)$ vs. projected radial distance from the location of the illumination source/expansion center ( $R$ in arcsec) in Fig. $3 \mathrm{~b}$. For each of the lobes and arc segments, linear fitting was done to characterize its proper motion. Upon fitting, we consider the following three plausible cases: (1) a linear expansion (linear fitting of $v_{\text {rad }}$ vs. $R$ anchored at the origin), (2) a constant-speed expansion (fitting by a horizontal line), and (3) a more general linear expansion (linear fitting with a non-zero intercept), and compared their Bayesian and Akaike information criteria (BIC and AIC)2

\footnotetext{
${ }^{2}$ In statistics, BIC/AIC are measures of the relative goodness of fit of a model which address issues of overfitting (i.e., a model with a larger number of parameters usually fits better) by penalizing models with a larger number of parameters.
} 
to identify the best-fit. Uncertainties in the projected speed and distances are roughly 5.0 mas $\mathrm{yr}^{-1}$ and $2^{\prime \prime}$, respectively. However, the relative spacing among individual structures within an ensemble remains the same and hence the slope of the linear fitting is robust.

The best-fit for the lobes yielded $v_{\text {rad }}=(2.56 \pm 0.15) \times R$ mas $\mathrm{yr}^{-1}$ or $(5.10 \pm$ $0.33) \times R \mathrm{~km} \mathrm{~s}^{-1}$ (the solid white line in the dark-gray zone), indicating that the lobes are expanding linearly with the dynamical age of $390 \pm 25 \mathrm{yr}$, which is roughly consistent with the previous results, $345 \pm 2$ and $266 \pm 40 \mathrm{yr}$ (Ueta et al. 2006 and Balick et al. 2012, respectively). Cox et al. (2000) measured the line of sight velocity of the tips of the CO bipolar lobes (coinciding with the optical lobe tip pair F1/F2 in their Figs. 2 and 3) as $6 \pm 1 \mathrm{~km} \mathrm{~s}^{-1}$ at $6^{\prime \prime} \pm 1^{\prime \prime}$ away from the center. By adopting the projected radial expansion velocity of $31 \pm 5 \mathrm{~km} \mathrm{~s}^{-1}$ at the tip of the $\mathrm{N}$ lobe, the inclination of the bipolar tips is computed as $11^{\circ} \pm 2^{\circ}$ and the deprojected bipolar outflow velocity as $32 \pm 5 \mathrm{~km} \mathrm{~s}^{-1}$. This outflow velocity is consistent with the medium-velocity component found in $\mathrm{CO}$ (Young et al. 1992; Yamamura et al. 1995).

The best-fit for the arc segments along the searchlight beams beyond the lobes turned out to be a constant-speed expansion at $v_{\text {rad }}=(5.28 \pm 0.17)$ mas $\mathrm{yr}^{-1}$ or $10.52 \pm 0.34 \mathrm{~km} \mathrm{~s}^{-1}$ (the solid black line in the light-gray zone), suggesting that the arcs have been ejected in an on-going basis and coasting away at a constant speed, as opposed to being formed altogether at the same time and expanding linearly. This is a new finding from the present analysis with a larger field of view with respect to the previous analysis by Balick et al. (2012), in which both growth patterns were found equally likely. A detection of the central CO shell of $1^{\prime \prime}$ radius expanding at around $10 \mathrm{~km} \mathrm{~s}^{-1}$ reported by Cox et al. (2000), therefore, may be related to the presence of these concentric arcs. If so, the generation of the concentric arcs appears to have been continuing even after the onset of the bipolar lobe expansion. 


\subsubsection{Radial Expansion of the Concentric Arcs}

To further alleviate the azimuthal degeneracy issue of the concentric arcs, we "unrolled" the edge-enhanced $I$ maps with respect to the adopted location of the expansion center to make polar $(r, \Theta)$ maps, where $r$ and $\Theta$ are the radial distance from the center and the position angle measured from $\mathrm{N}$, respectively. Then, we traced the projected radial motion of concentric arcs as a function of the position angle by performing the same correlational analysis only along the radial direction for each $1^{\circ}$ azimuthal arc segment as long as these arc segments register more than three- $\sigma$ of the background (Fig. 4a,b).

Fig. 4k shows the distribution of the projected radial velocities of these $1^{\circ}$ segments measured along 12 most prominent arcs. While uncertainties are relatively large owing to a large S/N discrepancy between the two-epoch maps, the present data suggest that the

projected radial velocities tend to (1) converge to a minimum of about $10 \mathrm{~km} \mathrm{~s}^{-1}$ toward the regions of the searchlight beams/bipolar axis, and (2) increase by a factor of about a few as moving toward the equatorial plane. While the scatterer in the measured radial velocities is large, the increasing tendency appears genuine.

If there were a well-defined azimuthal/latitudinal gradient in the radial expansion velocity field, it should have manifested itself in the overall structure of the concentric arcs - the arcs should have been elongated along the equatorial plane at least by a factor of a few. However, the arcs strikingly concentric. Therefore, it is most likely that there is a faster outflow (> $20-30 \mathrm{~km} \mathrm{~s}^{-1}$ ) in spatially restricted regions along the equatorial plane and that arc segments along the equatorial plane must have been blown out by such an outflow - the higher-velocity segments near the end of the observed arcs are most likely the arc edges being torn off by the suspected outflow.

Combined altogether, it appears that there are three distinct outflow components in the circumstellar environs of the Egg Nebula. There is a generally spherically symmetric 
steady outflow with a periodical modulation at about $10 \mathrm{~km} \mathrm{~s}^{-1}$, which is responsible for the concentric arcs. Based on the arc segments seen farthest from the center in the higher-S/N epoch 2 map, this periodic outflow appears to have continued at least for the past $\sim 5,500 \mathrm{yr}$ at the interval of $50-400 \mathrm{yr}$ at $420 \mathrm{pc}$.

This generally symmetric shell structure seems to be disrupted by two distinct outflow components, one along the bipolar axis and the other along the equatorial plane. It has already been shown that the bipolar lobes have been being excavated by a linear expansion for the past $300-400 \mathrm{yr}$ with the tip velocity being about $30 \mathrm{~km} \mathrm{~s}^{-1}$. Circumstantially, this linearly expanding outflow along the polar axis seems to have punched holes out of the central dust cocoon, through which most of the dust-scattered light streams out along the bipolar axis to illuminate the lobes. The present analysis of the concentric arcs has indicated the presence of another outflow component along the equatorial plane: its velocity appears to be greater than $20-30 \mathrm{~km} \mathrm{~s}^{-1}$ based on the ways that concentric arcs are being disrupted along the equatorial plane. Other observational details of this outflow including the mode and duration, however, remain unclear.

\subsection{Origins of the Outflow Components}

The apparent superposition of three outflow components in the Egg Nebula is dynamically very intriguing. While theoretical consideration of the latitudinal dependence of the velocity field has been rare (e.g., Dwarkadas, Chevalier, \& Blondin 1996), in studying

the influence of the central binary system to the AGB wind structure, Mastrodemos \& Morris (1999) numerically discovered that (1) the latitudinal dependence of the gas density is always accompanied by a latitudinal decline in the outflow velocity field, and (2) there can be a positive correlation between the latitudinal density and velocity contrasts (i.e., outflow velocity becomes faster where density is greater; their model 4), contrary to many 
dust-driven models that assume a velocity profile that increases toward the polar direction.

This means that the present case may provide rare observational evidence for such a colatitudinally-dependent outflow velocity field resulting from the egg-beater mass-loss modulation by the central binary system. While spiral modulation of mass-loss ejecta due to the central binary system has been seen around AGB stars for near pole-on cases (AFGL 3068; Mauron \& Huggins 2006, Mira; Maver et al. 2011, R Scl; Maercker et al. 2012), the present data may lend support for an edge-on case in which (1) the spiraling trails of mass-loss ejecta appear as concentric arcs, and (2) the colatitudinal velocity field promotes an equatorial outflow fast enough to disrupt the ejecta (e.g., Mastrodemos \& Morris 1999).

One issue in the present analysis is that the discrepancy in the signal strengths in the two-epoch data set (due to much lesser $\mathrm{S} / \mathrm{N}$ of epoch 1 data) resulted in rather large uncertainties in proper-motion measurements. At any rate, both theoretical and observational work is scarce at best to establish the connection between the concentric arcs and the binary modulation of stellar winds. Therefore, another epoch of HST observations to obtain data with a comparable $\mathrm{S} / \mathrm{N}$ to the epoch 2 data are extremely interesting in quantifying the colatitudinal dependence of the radial velocity field to be compared against the latest theoretical expectations (e.g., Mohamed \& Podsiadlowski 2011; Kim \& Taam 2012). Also, mapping these concentric arcs in CO at the comparable spatial resolution of the full ALMA is worthwhile (e.g., Maercker et al. 2012) to do understand kinematics between the gas and dust components of these outflows.

\section{Concluding Summary}

We performed differential proper-motion and radial-motion analyses on both the signature bipolar lobes and concentric arcs of the Egg Nebula using two-epoch optical data 
taken by the HST. Our method, based on (1) aligning two-epoch images using stationary background stars as spatial anchors, and (2) measuring the amount translational movement of specific local structures by a correlational method, is more robust than the previous analysis by Balick et al. (2012) that assumed symmetry in the shell expansion. Our findings are that;

1. the Egg Nebula is illuminated optically by a two-step mechanism, in which the direct starlight is first channeled into the bipolar cavities and then scattered off to the rest of the nebula,

2. there is a "dustsphere" around the central star $\left(1.0 \times 10^{3} \mathrm{AU}\right.$ by $1.6 \times 10^{3} \mathrm{AU}$ at 420 pc), most likely representing the surface of the central dust concentration at which the line-of-sight optical depth in the $V$ band becomes significantly larger than unity,

3. the bipolar lobes expand linearly for the past $390 \pm 25 \mathrm{yr}$, excavating into an otherwise symmetric concentric shells as found by the previous analyses (Ueta et al. 2006; Balick et al. 2012),

4. arc segments found along the searchlight beams beyond the bipolar lobes all move at similar projected radial velocity at $10.52 \pm 0.34 \mathrm{~km} \mathrm{~s}^{-1}$ for the past $\sim 5,500 \mathrm{yr}$,

5. the projected radial expansion velocity field of the concentric arcs shows an increasing trending toward the equatorial plane by a factor of a few, representing torn-off edges of the arcs disrupted by a fast equatorial outflow at $>20-30 \mathrm{~km} \mathrm{~s}^{-1}$, and

6. the present case may provide observational evidence for the binary modulation of stellar winds, as predicted by Mastrodemos \& Morris (1999), which generates the colatitudinal dependence in the radial expansion velocity field and the spiral-shocks of the mass-loss ejecta appearing concentric arcs in near edge-on orientations. 
Support for this work was provided by NASA/STScI through a grant HST-AR12157.01-A and by the University of Denver through a Professional Research Opportunities for Faculty (PROF) grant. Authors thank Dr. D. A. Weintraub for explaining his method to pinpoint the location of the illumination source in the imaging polarimetry data thoroughly to us. Authors also acknowledge Drs. Robert E. Stencel and Djazia Ladjal for their excellent comments which improved the work.

Facilities: HST (WFPC2,ACS/WFC). 


\section{REFERENCES}

Balick, B., Gomez, T., Vinković, D., Alcolea. J., Corradi, R. L. M., \& Frank, A. 2012, ApJ, 745,188

Biretta, J., \& McMaster, M. 1997, Instrument Science Report WFPC2 97-11 (Baltimore: STScI)

Cox, P., Lucas, R., Huggins, P. J., Forveille, T., Bachiller, R., Guilloteau, S., Maillard, J. P., \& Omont, A. 2000, A\&A, 353, L25

Currie, D. G., Dowling, D. M., \& Shaya, E. J., et al. 1996, AJ, 112, 1115

Dwarkadas, V. V., Chevalier, R. A., \& Blondin, J. M. 1996, ApJ, 457, 773

Fruchter, A., \& Sosey, M., et al. 2009, The MultiDrizzle Handbook, Ver. 3.0, (Baltimore: STScI)

Gonzaga, S., \& Biretta, J., et al. 2010, WFPC2 Data Handbook, Ver. 5.0 (Baltimore: STScI)

Gonzaga, S., et al., 2011, ACS Data Handbook, Ver. 6.0 (Baltimore: STScI)

Goto, M., Kobayashi, N., Terada, H., \& Tokunaga, A. T. 2002, ApJ, 572, 276

Hrivnak, B. J., Kwok, S. \& Su, K. Y. L. 2001, AJ, 121, 2775

Hrivnak, B. J., Lu, W., Maupin, R. E., \& Spitzbart, B. D. 2010, ApJ, 709, 1042

Kim, H., \& Taam, R. E. 2012, ApJ, 759, 59

Kwok, S. 1993, ARA\&A, 31, 63

Latter, W. B., Hora, J. L., Kelly, D. M., Deutsch, L. K., \& Maloney, P. R. 1993, AJ, 106, 260 
Maercker, M., Mohamed, S., Vlemmings, W. H. T., Ramstedt, S., Groenewegen, M. A. T., Humphreys, E., Kerschbaum, F., Lindqvist, M., Olofsson, H., Paladini, C., Wittkowski, M., de Gregorio-Monsalvo, I., \& Nyman, L.-A. 2012, Nature, 490, 7419

Mauron, N., \& Huggins, P. J. 2006, A\&A, 452, 257

Mayer, A., Jorissen, A., Kerschbaum, F., Mohamed, S., van Eck, S., Ottensamer, R., Blommaert, J. A. D. L., Decin, L., Groenewegen, M. A. T., Posch, Th., Vandenbussche, B., \& Waelkens, C. 2011, A\&A, 531, L4

Mohamed, S., \& Podsiadlowski, Ph. 2011, in ASP Conf. Ser. 445, Why Galaxies Care about AGB Stars II: Shining Examples and Common Inhabitants, eds. F. Kerschbaum et al. (San Francisco: ASP), 355

Mastrodemos, N., \& Morris, M. 1999, ApJ, 523, 357

Morse, J. A., Kellogg, J. R., Bally, J., Davidson, K., Balick, B., \& Ebbets, D. 2001, ApJ, 548, L207

Ney, E. P., Merrill, K. M., Becklin, E. E., Neugebauer, G., \& Wynn-Williams, C. G. 1975, ApJ, 198, L129

Oke, J. B. 1974, ApJS, 27, 21

Roberts, L. G. 1963, Lincoln Laboratory Technical Report, 315, 22 (Bostin: MIT)

Sahai, R., Hines, D. C., Kastner, J. H., et al. 1998, ApJ, 492, 163

Sahai, R., Trauger, J. T., Watson, A. M., et al. 1998, ApJ, 493, 301

Su, K. Y. L. 2004, in ASP Conf. Ser. 313, Asymmetrical Planetary Nebulae III, eds. M. Meixner et al. (San Francisco: ASP), 247 
Ueta, T., Murakawa, K., \& Meixner, M. 2005, AJ, 129, 1625

Ueta, T., Murakawa, K., \& Meixner, M. 2006, ApJ, 641, 1113

Ueta, T., Murakawa, K., \& Meixner, M. 2007, AJ, 133, 1345

Ueta, T., Murakawa, K., \& Meixner, M. 2012, ApJ, 756, 103

van Dokkum, P. G. 2001, PASP, 113, 1420

Van Winckel, H. 2003, ARA\&A, 41, 391

Weintraub, D. A., \& Kastner, J. H. 1993, ApJ, 411, 767

Weintraub, D. A., Kastner, J. H., Hines, D. C., \& Sahai, R. 2000, ApJ, 531, 401

Yamamura, I., Onaka, T., Kamijo, F., Deguchi, S., \& Ukita, N. 1995, ApJ, 439, L13

Young, K., Serabyn, G., Phillips, T. G., Knapp, G. R., Güsten, R., \& Schulz, A. 1992, ApJ, 385,265

This manuscript was prepared with the AAS LATEX macros v5.2.

Table 1. Two-Epoch Imaging-Polarimetry Observing Log of the Egg Nebula

\begin{tabular}{clcccc}
\hline \hline & & & & & \\
DATE & EXP TIME & & \\
& INSTRUMENT & FILTER & $($ sec $)$ & PID & DATASET ID \\
\hline & & & & & \\
1995 Jul 17 (epoch1) & WFPC2 & F606W/POLQ & 300.0 & 6221 & U2RC0301/2/3T \\
2002 Oct 16 (epoch2) & ACS/WFC & F606W/POLV & 999.0 & 9586 & J8GH5501/2/31 \\
\hline
\end{tabular}




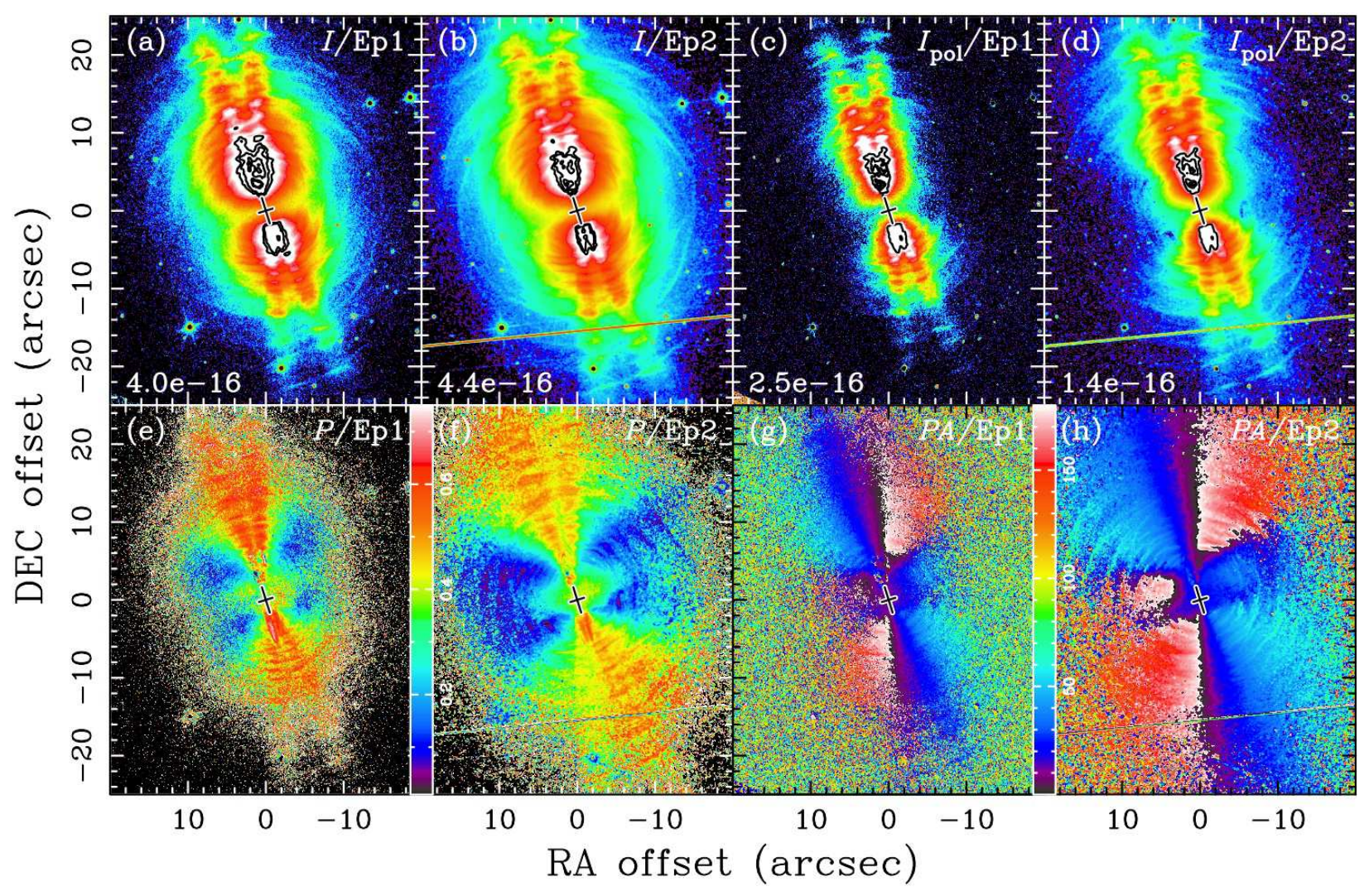

Fig. 1. - Two-epoch F606W images of the Egg Nebula in the total intensity, $I$ (a, b), linearpolarization-flux-only, $I_{\mathrm{pol}}(\mathrm{c}, \mathrm{d})$, polarization strength, $P(\mathrm{e}, \mathrm{f})$, and polarization angle, $P A$ (g, h). Each pair displays both epoch 1 (Ep1, left) and epoch 2 (Ep 2, right) images. The $I$ and $I_{\text {pol }}$ maps show surface brightnesses above $1 \sigma\left(4.3,1.0,6.1\right.$, and $0.8 \times 10^{-20} \mathrm{erg} \mathrm{cm}^{-2} \mathrm{~s}^{-1}$ $\AA^{-1} \mathrm{pix}^{-1}$, respectively for (a) to (d), in black) up to $1.0 \times 10^{-17} \mathrm{erg} \mathrm{cm}^{-2} \mathrm{~s}^{-1} \AA^{-1} \mathrm{pix}^{-1}$ (in white). Contours indicate 90, 50, 10, and $1 \%$ levels of the peak surface brightness, which is shown at lower-left in erg $\mathrm{cm}^{-2} \mathrm{~s}^{-1} \AA^{-1} \mathrm{pix}^{-1}$. The $P$ maps show polarization strengths in fraction as indicated in the color wedge, while the PA maps exhibit polarization vector angles in degrees measured from $\mathrm{W} /+x$ as indicated in the color wedge. All maps are centered at the position of the illumination source derived in our previous NICMOS differential propermotion analysis (Ueta et al. 2006), at which the plus symbol is to indicate the one- $\sigma$ error ellipse. 


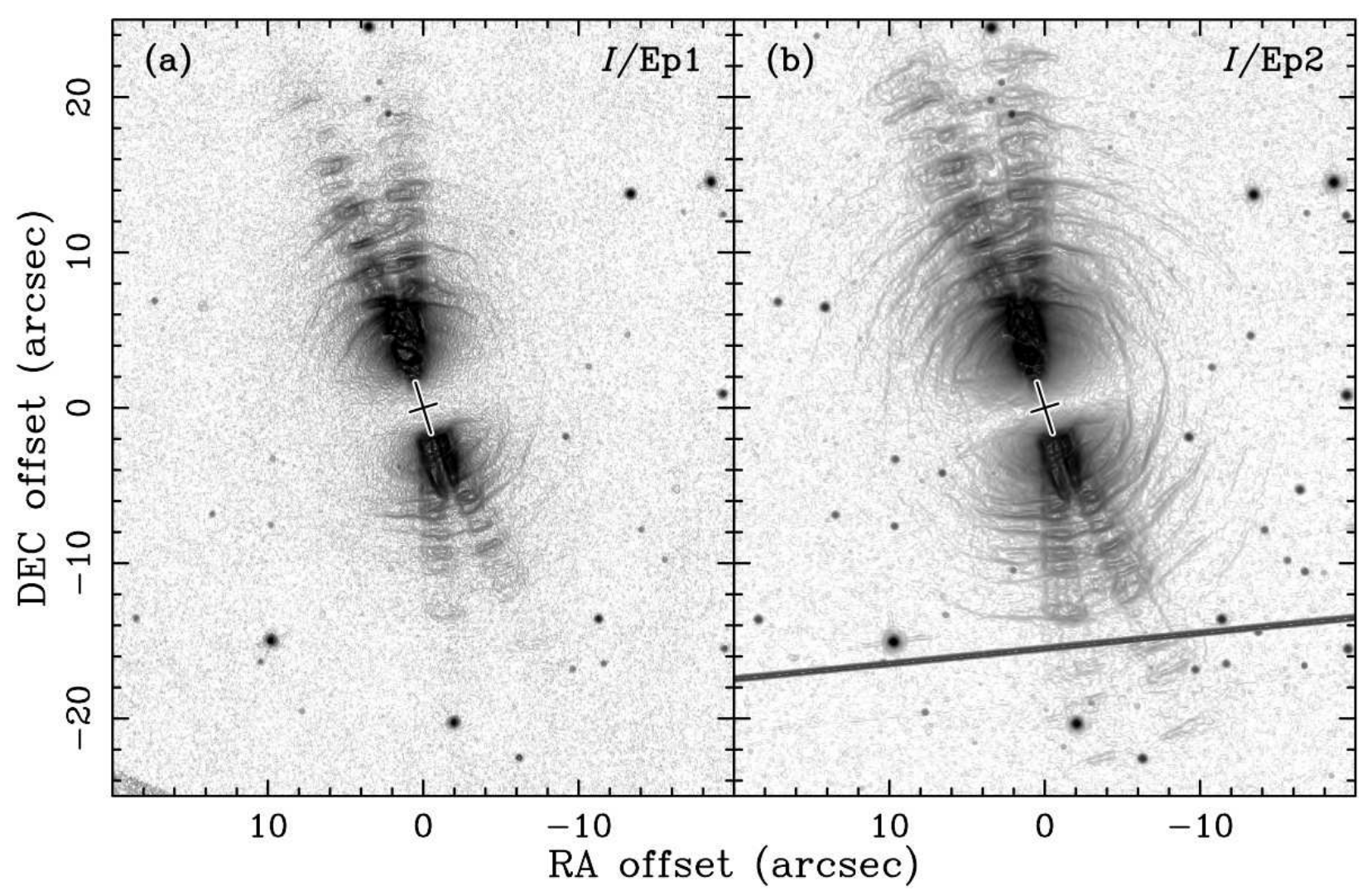

Fig. 2.- The edge-enhanced Stokes I maps of the Egg Nebula: (epoch 1 on left and epoch 2 on right). Image conventions follow Fig. 1. 

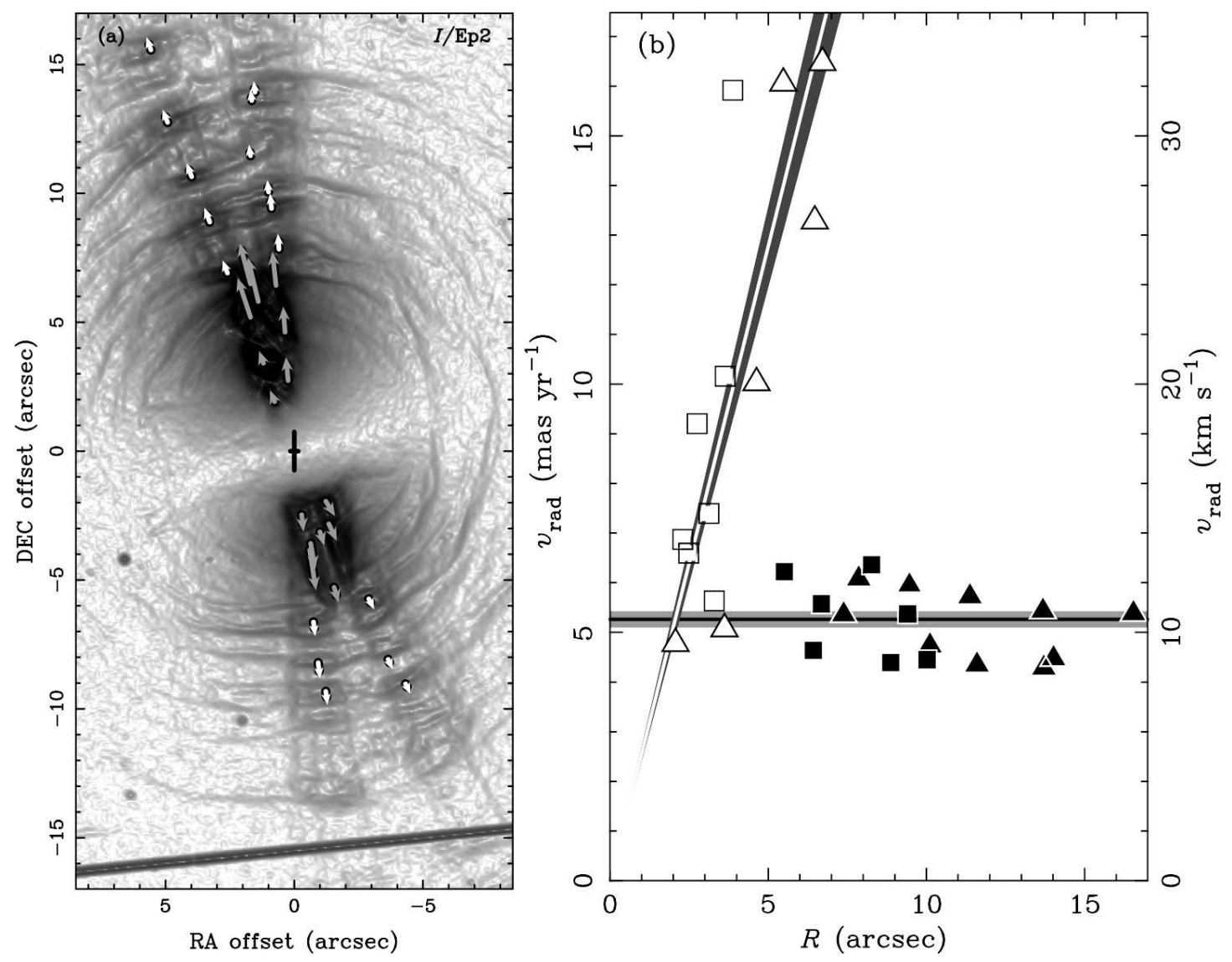

Fig. 3.- (a) The edge-enhanced $I$ map (of epoch 2 in grayscale) of the Egg Nebula overlaid with vectors of the projected radial component of the shell expansion. The length of the vectors depicts the relative velocity: gray vectors are of the lobes, while white vectors are of the arc segments. (b) The plot of $v_{\text {rad }}$ (projected radial component of the differential propermotion vector in $\operatorname{arcsec} \mathrm{yr}^{-1}$ and $\mathrm{km} \mathrm{s}^{-1}$ assuming $420 \mathrm{pc}$ ) vs. $R$ (projected radial distance from the expansion center in arcsec) for these structures. Symbols indicate structures of the lobe (open triangle - N lobe; open square - S lobe) and arc segments (filled triangle - arcs along the $\mathrm{N}$ searchlight beam; filled square - arcs along the S searchlight beam) The best-fit lines for the ensemble motion are shown as the white solid line with the gray zone of uncertainty (lobes) and black solid line with the light gray zone of uncertainty (arc segments). 


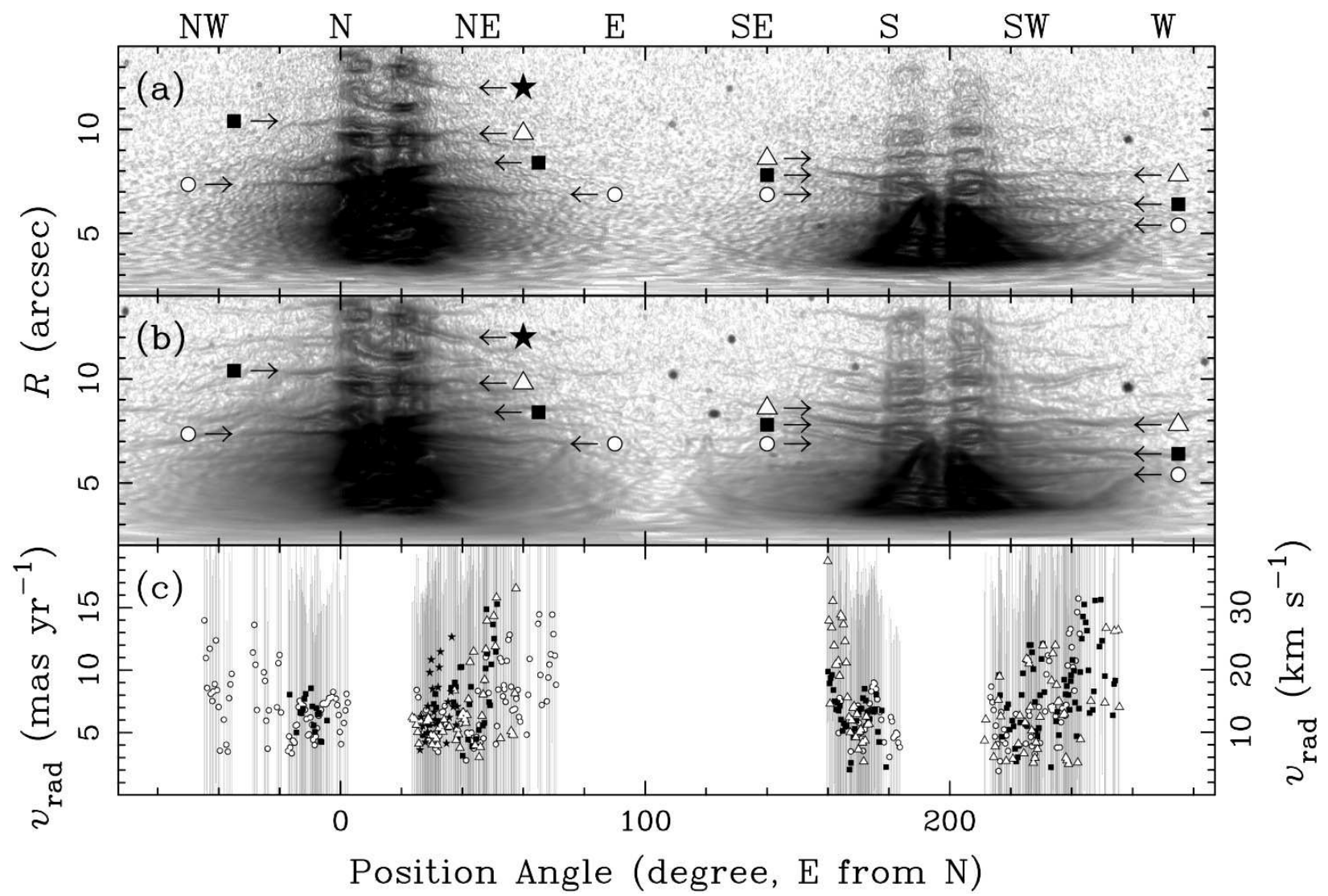

Fig. 4.- The polar edge-enhanced Stokes I polar maps of the Egg Nebula for epoch 1 (top) and epoch 2 (middle), with the distribution of measured projected radial expansion velocities in 12 most prominent arcs showing the apparent increasing trending of the radial velocity toward the equatorial plane (bottom). The polar angle is the position angle measured from $\mathrm{N}$ to E, with the orientations indicated above the top frame. Each symbol in the bottom frame represents a differential radial-motion measurement for each $1^{\circ}$ arc segment as a function of the position angle. The symbols also indicate the location of the arc in the polar maps. The expansion velocity, $v_{\mathrm{rad}}$, is given in mas $\mathrm{yr}^{-1}$ and $\mathrm{km} \mathrm{s}^{-1}$ (assuming $420 \mathrm{pc}$ ). Uncertainties of the radial velocity measurements are indicated by the vertical lines attached to each symbol. 
Table 2. Two-Epoch Aperture Photometry

\begin{tabular}{ccc}
\hline \hline & & $F_{\lambda}$ \\
Date & Aperture & $\left(10^{-12} \mathrm{erg} \mathrm{cm}^{-2} \mathrm{~s}^{-1} \AA^{-1}\right)$ \\
\hline & & \\
1995 Jul 17 & All & $1.85 \pm 0.02$ \\
& N Lobe & $1.38 \pm 0.01$ \\
& S Lobe & $0.47 \pm 0.01$ \\
& All & $1.18 \pm 0.01$ \\
& N Lobe & $0.90 \pm 0.01$ \\
& S Lobe & $0.27 \pm 0.01$
\end{tabular}

